\title{
Effects of milnacipran on binge eating - a pilot study
}

\author{
Shun'ichi Noma' \\ Teruhisa Uwatoko' \\ Haruka Yamamoto ${ }^{2}$ \\ Takuji Hayashi' \\ 'Department of Psychiatry, Graduate \\ School of Medicine, Kyoto University, \\ Kyoto, Japan; ${ }^{2}$ Department of \\ Psychiatry, Toyooka Hospital, Hyogo, \\ Japan
}

\begin{abstract}
Selective serotonin reuptake inhibitors and selective norepinephrine reuptake inhibitors are effective in the treatment of bulimia nervosa. There have been relatively few studies of the efficacy of specific serotonin and norepinephrine reuptake inhibitors in the treatment of eating disorders. Twenty-five outpatients with binge eating episodes, diagnosed as anorexia nervosa, binge-eating/purging type, bulimia nervosa/purging type, or bulimia nervosa/non-purging type, were treated with milnacipran and 20 patients completed the 8-week study. Symptom severity was evaluated using the Bulimic Investigatory Test, Edinburgh (BITE) self-rating scale before administration of milnacipran and after 1, 4, and 8 weeks treatment. The scores improved after 8 weeks, especially drive to, and regret for, binge eating. Milnacipran was more effective in patients without purging and in younger patients, while there was no difference in the efficacy of milnacipran among subtypes of eating disorders.
\end{abstract}

Keywords: milnacipran, specific serotonin and norepinephrine reuptake inhibitors, binge eating, vomiting, eating disorder, pharmacotherapy

\section{Introduction}

Many patients with eating disorders suffer from repeated episodes of binge eating, followed by inappropriate compensatory behaviors to prevent weight gain.

The discovery of disturbed brain serotonin activity in patients with bulimia nervosa (Jimerson et al 1992; Tauscher et al 2001) led to the use of selective serotonin reuptake inhibitors (SSRIs) for the treatment of patients with bulimic episodes even in the absence of depressive symptoms (Fichter et al 1996; Agras 1997; Romano et al 2002; Milano et al 2004). Disturbed noradrenergic function has also been implicated in bulimia nervosa (Brambilla 2001) and the efficacy of the selective norepinephrine reuptake inhibitor, reboxetine, in treating patients with bulimic episodes has been reported (El-Giamal et al 2000; Fassino et al 2004).

Thus specific serotonin and norepinephrine reuptake inhibitors (SNRIs) would be expected to be effective in the treatment of bulimic episodes in eating disorders and positive preliminary results have been reported with venlafaxine (Malhotra et al 2002) and duloxetine (Hazen and Fava 2006).

For milnacipran, the only SNRI currently available Japan, a pilot study (El-Giamal et al 2003) has reported the efficacy of milnacipran on purging binge eating.

We describe here a study investigating the efficacy of milnacipran in the treatment of binge eating in purging and non-purging patients.

\section{Methods}

\section{Participants}

Twenty-five outpatients suffering from eating disorders with binge eating (APA 1995), who were treated at the Kyoto University Hospital, Japan, from February 2004 through December 2006, were included in the study. No participant suffered from any 
psychiatric disorder other than eating disorders and none had previously taken any psychotropic medication.

The study, which was approved by the ethics committee of Kyoto University Hospital, was carried out in accordance with the Declaration of Helsinki and all participants gave their informed consent to participate in the study.

\section{Psychometric instruments}

Eating behavior including the frequency of binge eating and purging was assessed using the Bulimic Investigatory Test, Edinburgh (BITE) (Henderson and Freeman 1987). The BITE self-report questionnaire consists of 30 items of symptoms (BITE-symptom) and 6 items of severity (BITE-severity). The score of the BITE-symptom is the total number of "no" answers in items 1, 13, 21, 23, and 31 and "yes" answers in the other items. The BITE-severity score is the number of 6 items present (fasting, taking diet pills, diuretics, and laxatives, self-induced vomiting, and binge eating episodes). A Japanese version of the BITE and its validation have been published (Nakai et al 1998). The prevalence of depressive and anxious symptoms was rated using the Hamilton Depression Rating Scale (HAM-D) (Hamilton 1960) and the Hamilton Anxiety Rating Scale (HAM-A) (Hamilton 1959), respectively. The general condition and the degree of improvement was assessed using the Clinical Global Impression (CGI) (National Institute of Mental Health 1976).

\section{Procedures}

All subjects were assessed 4 times during the 8-week study using BITE, HAM-D, HAM-A, and CGI - at baseline prior to milnacipran administration and following 1 week, 4 weeks, and 8 weeks of drug administration.

The dosing schedule was not fixed in advance but determined for each patient on the basis of their positive (symptom reduction) and negative (side-effects) reaction to the drug. Milnacipran was taken twice daily, just after getting up and just before going to bed in order to reduce the risk of disgorging the milnacipran tablets during self-induced vomiting. Mosapride, $15 \mathrm{mg} / \mathrm{day}$, could be given to the patients with nausea, if necessary.

Standard supportive psychotherapy was provided for all patients but no cognitive behavioral therapy was undertaken.

\section{Analysis}

Differences between baseline and post-treatment values were assessed by Student's paired t-test or the Wilcoxon test. Tukey's test was used for multiple comparisons. Correlations were tested using Pearson's correlation coefficient test. A one-way ANOVA was used to test differences in characteristics among the subgroups of eating disorders.

\section{Results}

\section{Characteristics of the subjects}

Of the 25 patients recruited, 5 withdrew before the end of the study. Two patients chose to stop treatment in favor of psychotherapy without reporting any side-effects. Three patients complained of severe nausea, which was considered probably due to milnacipran and disappeared upon discontinuation of the drug.

Eating disorders with binge eating can be categorized as anorexia nervosa, binge-eating/purging type (ANBP), bulimia nervosa/purging type (BNP), bulimia nervosa/nonpurging type (BNNP), or binge eating disorder (BED) (APA 1995). The characteristics of the 20 subjects who completed the 8-week study are shown in Table 1 . Subjects were diagnosed with ANBP, BNP, and BNNP subtypes of eating disorders. BED was not found.

There were no significant differences in characteristics between patients with different subtypes of eating disorder with the exception of weight and body mass index (BMI). One of the 6 patients with anorexia nervosa had no purging

Table I Patient characteristics

\begin{tabular}{lllll}
\hline Number & Total & ANBP & BNP & \multicolumn{1}{c}{ BNNP } \\
$\mathbf{7}$ & $\mathbf{2 0}$ & $\mathbf{6}$ & $\mathbf{7}$ & $22.9 \pm 4.5$ \\
\hline Age (years) & $22.4 \pm 4.1$ & $22.17 \pm 2.71$ & $22.0 \pm 5.3$ & $156.3 \pm 6.1$ \\
Height $(\mathrm{cm})$ & $156.7 \pm 5.6$ & $158.3 \pm 7.7$ & $155.6 \pm 4.7$ & $57.6 \pm 17.4$ \\
Weight $(\mathrm{kg})$ & $48.3 \pm 13.5$ & $37.1 \pm 5.5^{*}$ & $48.5 \pm 4.7$ & $23.5 \pm 6.2$ \\
Body Mass Index $\left(\mathrm{kg} / \mathrm{m}^{2}\right)$ & $19.7 \pm 5.2$ & $14.7 \pm 1.3^{* *}$ & $20.0 \pm 1.8$ & $76.1 \pm 72.9$ \\
Duration of eating disorders (months) & $62.9 \pm 58.6$ & $60.2 \pm 41.2$ & $52.0 \pm 61.6$ & $27.6 \pm 42.4$ \\
Duration of binge episodes (months) & $38.9 \pm 46.1$ & $45.8 \pm 39.6$ & $44.1 \pm 58.3$ & $11.0 \pm 23.2$ \\
Length of previous treatment (months) & $21.7 \pm 30.4$ & $36.0 \pm 32.3$ & & $20.0 \pm 34.4$ \\
\hline
\end{tabular}

Values are given as mean $\pm \mathrm{SD}$.

Notes: ${ }^{*} p=0.019$; ${ }^{*} p=0.009$ compared to BNNP.

Abbreviations: ANBP, binge-eating/purging type; BNP, bulimia nervosa/purging type; BNNP, bulimia nervosa/non-purging type. 
episodes. There were thus 12 purgers who vomited regularly, and 8 non-purgers who did not.

Milnacipran was administered initially at doses from 15 to 75 (mean \pm SD: $37.0 \pm 14.1$ ) mg daily increasing to 25 to $100(60.0 \pm 22.0) \mathrm{mg}$ daily after 8 weeks. The target dose of $100 \mathrm{mg} /$ day was achieved in only 3 patients. Ten patients reported some adverse events, judged to be possibly related to milnacipran, and 7 patients refused dose increases because of fear of possible adverse effects. Of 10 patients reporting adverse events, 8 patients complained of nausea, 2 of dizziness, and 1 a slight fever (1 patient complained of both nausea and dizziness).

\section{Improvement of BITE, HAM-D, HAM-A and $\mathrm{CGI}$ scores}

Changes in BITE, HAM-D,HAM-A, and CGI scores are shown in Figure 1. Significant improvements were found after 1 week on HAM-D, HAM-A, CGI-severity, and CGI-improvement scores and after 4 weeks on the
BITE-symptom scores. At 8 weeks, BITE-symptom, HAM-D, HAM-A, CGI-severity, and CGI-improvement scores were all significantly improved from baseline. In addition there was a trend $(p=0.065)$ for improvement on the BITE-severity score at 8 weeks. There was no correlation between the improvement of BITE scores after 8 weeks and the final dose of milnacipran.

Six items of the BITE-symptom were significantly improved after 8 weeks (Table 2). Significant reduction of these items may be interpreted as a reduction in the drive to, and regret for, binge eating. In addition there was a trend for a reduction in the frequency of binge eating $(p=0.086)$ and the frequency of vomiting $(p=0.083)$.

\section{BITE score and HAM-D and HAM-A scores}

There was a significant positive correlation $(\mathrm{r}=0.481$, $\mathrm{p}=0.032$ ) between the improvement of the BITE-symptom scores and HAM-A scores after 8 weeks but not between

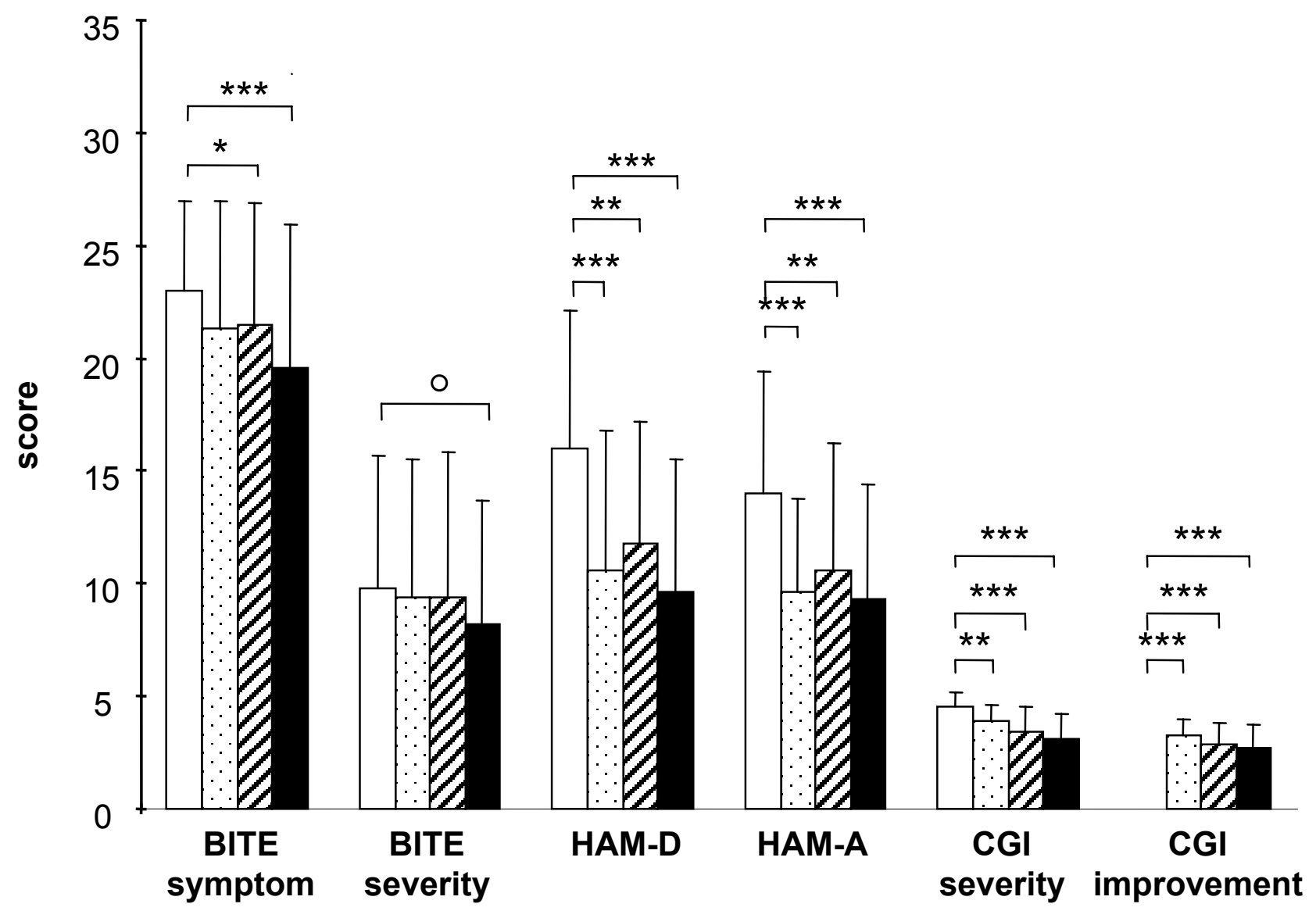

Figure I Changes in BITE, HAM-D, HAM-A, and CGI scores. Open columns = baseline - just before first milnacipran administration; Stippled columns = after I week administration of milnacipran; Striped columns = after 4 weeks' administration of milnacipran; Solid columns $=8$ weeks' administration of milnacipran. Notes: ${ }^{\circ} \mathrm{p}<0.1 ; * * \mathrm{p}<0.05 ;{ }^{* * *} \mathrm{p}<0.01$; ${ }^{* * * *} \mathrm{p}<0.00$ I between values indicated.

Abbreviations: BITE, Bulimic Investigatory Test, Edinburgh; CGI, Clinical Global Impression; HAM-D, Hamilton Depression Rating Scale; HAM-A, Hamilton Anxiety Rating Scale. 
Table 2 Items of the Bulimic Investigatory Test, Edinburgh (BITE)-symptom scale on which the frequency of the answer "yes" ("no" in item 13) was significantly reduced during the 8 week study

\begin{tabular}{llll}
\hline Item number & Item content & Reduction from baseline & P \\
\hline 3. & Do you feel a failure if you break your diet once? & $25 \%$ & 0.025 \\
9. & Would you say that food dominated your life? & $20 \%$ & 0.046 \\
I0. & Do you ever eat and eat until you are stopped by physical discomfort? & $35 \%$ & 0.008 \\
I3. & Can you always stop eating when you want to? & $35 \%$ & 0.020 \\
I4. & Do you ever experience overpowering urges to eat and eat and eat? & $25 \%$ & 0.025 \\
20. & Do you turn to food for comfort? & $20 \%$ & 0.046 \\
\hline
\end{tabular}

The other items of the scale were not significantly modified from baseline.

the improvement of BITE-symptom scores and HAM-D scores. There was no correlation between improvement of BITE scores after 8 weeks and HAM-D and HAM-A scores at baseline.

\section{BITE score improvement and eating disorder subgroups}

With the exception of a significant difference in the improvement of BITE-symptom scores $(p=0.041)$ after 1 week, there were no differences at any time point in the degree of improvement of BITE-symptom or BITE-severity scores in patients with anorexia nervosa and those with bulimia nervosa.

At 8 weeks, non-purging patients showed a significantly greater improvement $(\mathrm{p}=0.017)$ in BITE-symptom score than purging patients. BITE-severity scores, however, were not significantly different.

\section{BITE score improvement and patient background}

Age was negatively correlated with bite-severity score at 8 weeks $(r=-0.478, p=0.033)$. No other correlations were found with BITE scores and weight, BMI, duration of eating disorders, duration of binge episodes, or length of treatment.

\section{Discussion}

Pathological eating attitudes were significantly improved after 4 weeks' milnacipran administration and there was a trend for the frequencies of binge eating and self-induced vomiting to decrease after 8 weeks. This is globally similar to the results reported previously in 16 patients with bulimia nervosa (El-Giamal et al 2003).

Milnacipran significantly reduced the drive to binge and the regret for binge eating, while pathological ideas and behaviors common to all patients with eating disorders, such as obsession about diet, fear of obesity, eating in hiding, did not change. This suggests that milnacipran does not improve the fundamental psychopathology of eating disorders but only reduces the drive to binge eating.

Depressive mood and anxiety were significantly improved after 1 week. In view of the short treatment period, however, the possibility of a placebo effect cannot be ruled out. The significant correlation between HAM-A and BITE-symptom scores suggests an effect of improved eating attitudes on anxiety (or possibly the other way round). Eating attitudes and depressive state, however, appear to be unrelated.

The reduction of the frequencies of binge eating and vomiting after 8 weeks was not statistically significant. The BITE-severity is a relatively crude measure and more precise results may have been obtained if patients had recorded details of their own eating attitudes in addition to using the BITE-severity.

Doses of antidepressants, especially SSRI, higher than those typically used for depression are thought to be required for the effective treatment of bulimia (Carter et al 2003). The mean dose of milnacipran that the patients took in this study (60 mg per day after 8 weeks) was, however, similar to the mean antidepressant dose in Japan. Although there was no significant correlation between the degree of improvement in eating attitudes and the dose of milnacipran (possibly as a result of the small sample size), it is possible that higher doses may have been more effective. In future studies it may be useful to consider increasing the dose of milnacipran up to 150 or $200 \mathrm{mg} /$ day in patients whose bulimic episodes are not sufficiently improved, as long as the patient can tolerate these doses.

Eleven of the 25 (44\%) participants in this study and 8 of the $20(40 \%)$ patients who completed the study complained of nausea to some extent. While milnacipran has been reported to produce fewer gastrointestinal side-effects than SSRIs, nausea is a primary side-effect whose severity can be reduced by slow titration (Lopez-Ibor et al 1996). The free 
titration allowed by the protocol and the use of mosapride may explain why only 3 patients ( $9 \%$ ) dropped out because of nausea. Patients with eating disorders are particularly sensitive to gastrointestinal symptoms and side-effects and it is important to start with low doses of milnacipran and increase the dose by slow titration, using anti-emetic medication where necessary.

Eating attitudes were improved to a greater extent in patients without purging behavior. This may be related to loss of the test drug during vomiting although administration times were chosen to reduce this effect.

Milnacipran appears to be effective in the treatment of binge eating disorder, which is present in almost half of all the patients with eating disorders (Fairburn and Harrisson 2003). Milnacipran should be tried in patients with binge eating episodes even if they have suffered for a long time because there its efficacy does not seem to be modified by duration of eating disorders or previous treatment.

The present study suffered from a number of weaknesses, principally its relatively small sample size, the lack of a control group, and the heterogeneity of the sample population. In addition the study followed the patients for only 8 weeks. The dosing schedule resulted in the use of relatively low doses of milnacipran. While flexible dosing according to patients' needs reflects routine clinical use, it adds to the heterogeneity of the sample. Although theoretically the use of higher doses (up to $200 \mathrm{mg} /$ day) may have improved the outcome, the inability or refusal of most patients to accept higher doses reflects the real-world limitations of dose escalation in this sensitive population. In a future study it would be helpful to monitor milnacipran plasma levels in order to be sure that vomiting did not reduce drug absorption. Finally, although the BITE score is a useful parameter it is rather crude and the use of more detailed measures of eating behavior would ensure a more sensitive measure of efficacy.

\section{Conclusion}

The results of this relatively small open study suggest that milnacipran may be effective in the treatment of eating disorders with binge eating episodes, reducing drive to binge and the regret for binge eating and, to a lesser extent, the frequencies of binge eating and vomiting. Milnacipran appears to have similar efficacy in all subtypes of eating disorders studied although patients who purged responded less well. A longer-term, placebo-controlled, double-blind study of milnacipran in eating disorders with binge eating would appear to be justified.

\section{Acknowledgments}

We thank Dr Yoshikatsu Nakai and Dr Seiji Hamagaki for their helpful advice and comments and Dr Mike Briley for his help in correcting the manuscript.

After completion of the study and its analysis, Asahi-Kasai Pharma, manufacturers of milnacipran in Japan, accepted to support the publication of the study with an unconditional educational grant. They played no role in the interpretation of the data, in the writing of the manuscript, or in its submission for publication.

\section{References}

Agras WS. 1997. Pharmacotherapy of bulimia nervosa and binge eating disorder: longer-term outcomes. Psychopharmacol Bull, 33:433-6.

American Psychiatric Association. 1995. Diagnostic and Statistical Manual of Mental Disorders, 4th Ed. Washington, DC: American Psychiatric Association.

Brambilla F. 2001. Aetiopathogenesis and pathophysiology of bulimia nervosa: biological bases and implications for treatment. CNS Drugs, 15:119-36.

Carter WP, Hudson JI, Lalonde JK, et al. 2003. Pharmacologic treatment of binge eating disorder. Int J Eat Disord, 34:S74-S88.

Clerc G, Milnacipran/Fluvoxamine Study group. 2001. Antidepressant efficacy and tolerability of milnacipran, a dual serotonin and noradrenaline reuptake inhibitor: a comparison with fluvoxamine. Int Clin Psychopharmacol, 16:145-51.

El-Giamal N, de Zwaan M, Bailer U, et al. 2000. Reboxetine in the treatment of bulimia nervosa: a report of seven cases. Int Clin Psychopharmacol, 15:351-6.

El-Giamal N, de Zwaan M, Bailer U, et al. 2003. Milnacipran in the treatment of bulimia nervosa: a report of 16 cases. Eur Neuropsychopharmacol, 13:73-9.

Fairburn CG, Harrison PJ. 2003. Eating disorders. Lancet, 361:407-16.

Fassino S, Daga GA, Boggio S, et al. 2004. Use of reboxetine in bulimia nervosa: a pilot study. J Psychopharmacol, 18:423-8.

Fichter MM, Kruger R, Rief W, et al. 1996. Fluvoxamine in prevention of relapse in bulimia nervosa: effects on eating-specific psychopathology. J Clin Psychopharmacol, 16:9-18.

First MB, Spitzer RL, Gibbon M et al. 1997. Structured Clinical Interview for DSM-IV axis 1 Disorders. Patient edition (SCID-I/P, version 2.0). New York: Biometric Research, New York State Psychiatric Institute.

Fukuchi T, Kanemoto K. 2002. Differential effects of milnacipran and fluvoxamine, especially in patients with severe depression and agitated depression: a case-control study. Int Clin Psychopharmacol, 17:53-8.

Hamilton M. 1959. The assessment of anxiety states by rating. Br J Med Psychol, 32:50-5.

Hamilton M. 1969. A rating scale for depression. J Neurol Neurosurg Psychiatry, 23:56-62.

Hazen E, Fava M. 2006. Successful treatment with duloxetine in a case of treatment refractory bulimia nervosa: a case report. J Psychopharmacol, 20:723-4.

Henderson M, Freeman CP. 1987. A self-rating scale for bulimia, the 'BITE' Br J Psychiatry, 150:18-24.

Jimerson DC, Lesem MD, Kaye WH, et al. 1992. Low serotonin and dopamine metabolite concentrations in cerebrospinal fluid from bulimic patients with frequent binge episodes. Arch Gen Psychiatry, 49:132-8.

Lopez-Ibor J, Guelfi JD, Pletan Y, et al. 1996. Milnacipran and selective serotonin reuptake inhibitors in major depression. Int Clin Psychopharmacol, 11(suppl 4):41-6.

Malhotra S, King KH, Welge JA, et al. 2002. Venlafaxine treatment of binge-eating disorder associated with obesity: a series of 35 patients. J Clin Psychiatry, 63:802-6. 
Milano W, Petrella C, Sabatino C et al. 2004. Treatment of bulimia nervosa with sertraline: a randomized controlled trial. Adv Ther, 21:232-7.

Nakai Y, Hamagaki S, Takagi R. 1998. The validity of Bulimic Investigatory Test, Edinburgh (BITE) and the survey of bulimia nervosa. Seishin igaku, 40:711-16.

National Institute of Mental Health. 1976. Clinical Global Impressions. In: Guy W ed. ECDEU Assessment Manual for Psychopharmacology, Revised Ed. Rockville, MD: NIMH, pp. 217-22.

Romano SJ, Halmi KA, Sarkar NP, et al. 2002. A placebo-controlled study of fluoxetine in continued treatment of bulimia nervosa after successful acute fluoxetine treatment. Am J Psychiatry, 159:96-102.

Tauscher J, Pirker W, Willeit M, et al. 2001. [123I] B-CTI and single photon emission computed tomography reveal reduced brain serotonin transporter availability in bulimia nervosa. Biol Psychiatry, 49:326-32. 